\title{
ESTUDIO PILOTO EN CUIDADORES DE PACIENTES CON ENFERMEDADES NEUROLÓGICAS, SOBRE EL SIGNIFICADO Y CONOCIMIENTO DE CUIDADOS PALIATIVOS
}

\begin{abstract}
Zoila Trujillo de los Santos ${ }^{1}$, Francisco Paz-Rodríguez ${ }^{1}$, María Alejandra Sánchez Guzmán ${ }^{1}$, Guadalupe Nava García ${ }^{1}$, Patricia Zamora R. ${ }^{1}$, Claudia García Pastrana ${ }^{1}$, Adriana Ochoa Morales ${ }^{1}$, María del Refugio Medina Durán ${ }^{1}$

Resumen: Objetivo. Describir el significado que atribuyen cuidadores a los cuidados paliativos (CPS), así como su preferencia respecto a informar al paciente y su satisfacción con el papel de cuidador. Metodología. Estudio descriptivo transversal, muestra intencional de 40 cuidadores de enfermos neurológicos con necesidad de CPS. Se aplicó un cuestionario de conocimientos sobre el tema y sobrecarga. Se realizó análisis descriptivo-comparativo con un intervalo de confianza de $95 \%$ ( $p<0.05)$. Resultados. Pacientes: 52,6\% hombres, edad promedio de 57,9 años, tiempo de enfermedad: 5 años, 2 hijos en promedio. Cuidadores: $77,5 \%$ mujeres, $25,0 \%$ cónyuges. Edad promedio: 49.3 ańos, proporcionan 18,5 horas de cuidado diario, 62,5\% tenía estudios de secundaria o superiores, $40 \%$ son cuidadores únicos, $16,7 \%$ no cuenta con redes de apoyo y $42,5 \%$ presenta sobrecarga alta. Un 70\% no tenía información previa sobre CPS. Significado: 50\% mencionó "dar ayuda" (física o emocional), 20\% "mejorar la calidad de vida". El 87,5\% desearía ser informado con detalle si ellos fueran los pacientes, $86,1 \%$ quisiera ser informado por el médico y $62,5 \%$ se mostró a favor de que se le informara con detalle a su familiar. Conclusiones. Es importante difundir los CPS en cuidadores y estimular el derecho de los pacientes a recibir información sobre el pronóstico de su enfermedad.
\end{abstract}

Palabras clave: cuidados paliativos, medicina paliativa, percepción, sobrecarga

Pilot study with caregivers attending patients with neurologic diseases, about the meaning and knowledge of palliative care

Abstract: Aim. To describe the meaning attributed to palliative care (PC) by caregivers, as well as their preference with respect to informing the patient and their satisfaction in their caring role. Methodology: Study descriptive transversal, sample intended of 40 caregivers of neurologic patients needing PC. A questionnaire about knowledge of the topic and their burnout status was applied. A descriptive-comparative analysis was carried out with $95 \%(\mathrm{p}<0.05)$ confidence interval. Results: Patients: $52.6 \%$ men, average age 57.9 years, duration of disease: 5 years, average of 2 children. Caregivers: $77.5 \%$ women, $25.0 \%$ married, average age: 49.3 years, daily caring 18.5 hours, $62.5 \%$ with high School or university studies, $40 \%$ alone as caregivers, $16.7 \%$ do not count with support nets and $42.5 \%$ present high burn out status. Of them $70 \%$ did not have previous information about PC. Meaning: 50\% mentioned "to give help" (physical or emotional), 20\% "to enhance quality of life". $87.5 \%$ would like to be informed with detail if they were the patients, $86.1 \%$ would like to be informed by the physician and $62.5 \%$ were in favor to inform with detail to their family member. Conclusions: It is important to disseminate PC to caregivers and to stimulate the right of patients to receive information about the prognosis of their disease.

Key words: palliative care, palliative medicine, perception, burnout

Estudo piloto em cuidadores de pacientes com enfermidades neurológicas, sobre o significado e conhecimento de cuidados paliativos

Resumo: Objetivo. Descrever o significado que atribuem cuidadores aos cuidados paliativos (CPS), assim como sua preferência com respeito a informar ao paciente e sua satisfação com o papel de cuidador. Metodologia. Estudo descritivo transversal, amostra intencional de 40 cuidadores de enfermos neurológicos com necessidade de CPS. Aplicou-se um questionário de conhecimentos sobre o tema e sobrecarga. Realizou-se análise descritivo-comparativa com um intervalo de confiança de $95 \%$ ( $\mathrm{p}<0.05)$. Resultados. Pacientes: 52,6\% homens, idade média de 57,9 anos, tempo de enfermidade: 5 anos, 2 filhos em média. Cuidadores: 77,5\% mulheres, 25,0\% cônjuges. Idade média: 49.3 anos, proporcionam 18,5 horas de cuidado diário, 62,5\% tinham estudos secundários ou superiores, $40 \%$ são cuidadores únicos, $16,7 \%$ não contam com redes de apoio e $42,5 \%$ apresentam sobrecarga alta. 70\% não tinham informação prévia sobre CPS. Significado: $50 \%$ mencionaram "dar ajuda" (física ou emocional), $20 \%$ "melhorar a qualidade de vida". $87,5 \%$ desejariam ser informados com detalhe se eles fossem pacientes, $86,1 \%$ quiseram ser informados pelo médico e $62,5 \%$ se mostraram a favor de que fossem informados com detalhes um seu familiar. Conclusóes. É importante difundir os CPS em cuidadores e estimular o direito dos pacientes em receber informação sobre o prognóstico de sua enfermidade.

Palavras-chave: cuidados paliativos, medicina paliativa, percepção, sobrecarga

\footnotetext{
${ }^{1}$ Programa de Redes de Apoyo para Cuidados Paliativos de Pacientes Neurológicos (PRACUP), México D.F., México Correspondencia: fpaz@innn.edu.mx
} 


\section{Introducción}

El envejecimiento poblacional ha traído consigo cambios en el patrón epidemiológico, con incremento notable de enfermedades crónicas, especialmente neurodegenerativas, como Parkinson, demencias, neoplasias cerebrales $y$, aunque no degenerativa, la enfermedad vascular cerebral(1). Con los avances tecnológicos, tanto en el diagnóstico como en el tratamiento, se ha logrado mayor supervivencia; sin embargo, los pacientes con enfermedades neurológicas enfrentan cada vez más una terminalidad (la etapa final de la enfermedad en la cual el pronóstico de supervivencia no es mayor a seis meses) poco atendida o muy limitada, de ahí que la necesidad de Cuidados Paliativos (CPS) se incremente día con día.

En este contexto, en México, la implementación de los CPS ha tenido un desarrollo lento y heterogéneo. Inicialmente concebidas como "clínicas del dolor", poco a poco se fueron integrando servicios o programas de CPS. La formación de profesionales dedicados a la disciplina paliati$\mathrm{va}(2,3)$ ha sido todavía más heterogénea y, de manera natural, los anestesiólogos han derivado en "algólogos", que posteriormente se han integrado a dichos servicios. La oferta para formación de paliativistas se centra en diplomados, existiendo pocos cursos de especialización para médicos y enfermeras paliativistas(4).

Atendiendo a las indicaciones de la Organización Mundial de la Salud(5) (1990), en el sentido de que la implementación de los Cuidados Paliativos es una necesidad indubitable y debe formar parte de una política de salud, en México se hizo una reforma a la Ley General de Salud(6) que instruye sobre la implementación de CPS de manera integral en todos los niveles de atención.

La actitud negativa hacia los CPS de muchos médicos en nuestro país proviene de la insuficiente formación en este sentido en el currículo profesional, así como la permanencia de actitudes paternalistas, influenciadas por nuestros propios patrones culturales $(7,8)$. Informar a los pacientes sobre el diagnóstico de enfermedades neurodegenerativas (esclerosis lateral amiotrófica, esclerosis múltiple, demencia o tumores cerebrales), cuya evolución y pronóstico es malo en un tiempo de- terminado, puede ser cuestionable para muchos médicos y prefieren matizar la información o darla en primera instancia a la familia, contraviniendo los principios sobre la autonomía y derecho del paciente a ser informado(9-11).

En México es aún poca la cobertura de la atención paliativa en el medio hospitalario. Un buen porcentaje de enfermos terminales debe ser atendido y cuidado en su medio familiar, sin que se le ofrezca la extensión del cuidado paliativo profesional, razón por la cual, la carga del cuidado puede ser severa, tanto en lo físico como en lo económico y social(4). Se ha documentado la sobrecarga y agobio en los cuidadores de pacientes con algunas enfermedades crónicas y neurodegenerativas (Parkinson, demencia, esclerosis lateral amiotrófica, entre otras) (12,13), sin embargo, muy poco se ha investigado en los cuidados paliativos para pacientes con estos y otros padecimientos neurológicos.

En el estudio realizado por Luxardo et al.(14) con 50 cuidadores de pacientes en CPS, sobre el perfil y el papel en su tarea de cuidadores, destaca la importancia de la ansiedad que genera la pérdida de apetito y la falta de actividad de los enfermos, tema que impacta la percepción de competencia de los cuidadores. Por su parte, Cuesta et al.(15), en su trabajo con 95 cuidadores de pacientes en situación terminal, investigaron la preferencia de informar el diagnóstico al paciente. El 60\% opinó que era necesario informar con detalle al paciente, aumentando esta preferencia a un $80 \%$ en el caso de que ellos fueran los pacientes.

El objetivo del presente estudio piloto es describir el significado que atribuyen los cuidadores a los cuidados paliativos, así como sus preferencias respecto de informar a los pacientes y su satisfacción con el cuidado brindado.

\section{Metodología}

Estudio descriptivo transversal, en una muestra intencional, realizado en el Programa de Redes de Apoyo para Cuidados Paliativos de pacientes neurológicos (PRACUP) del Instituto Nacional de Neurología y Neurocirugía (INNN), entre diciembre de 2012 y febrero de 2013. Se entrevistó a 40 cuidadores de pacientes neurológicos con 
necesidad de cuidados paliativos (entendiéndose como aquel paciente cuya enfermedad tuviera pronóstico fatal y sin posibilidades de continuar tratamiento curativo, ameritando solo tratamiento sintomático y de confort, esencia del cuidado paliativo). Los criterios de inclusión fueron: ser cuidador de un paciente terminal (definido como la etapa de la enfermedad en la que no existe respuesta a tratamiento curativo y existen múltiples síntomas físicos y psicológicos que causan sufrimiento al paciente y a la familia, y cuyo pronóstico es fatal a un plazo no mayor a seis meses); ser mayor de edad y manifestar ser el cuidador del paciente; no percibir salario por las tareas de cuidado; acudir a consulta o tener a su paciente en hospitalización durante el periodo de estudio y que el entrevistado no manifestara problemas de comprensión o comunicación. Se consideró cuidador a un familiar directo del paciente (pareja, hijos, padres, hermanos) involucrado en su cuidado. En el caso de que el paciente contará con más de un cuidador, se entrevistó al más involucrado en los cuidados.

El equipo de investigación estuvo conformado por integrantes del PRACUP y dos investigadores externos (Departamento de Neuropsicología y Grupos de Apoyo y Laboratorio de Violencia). De una lista, obtenida en el PRACUP, de pacientes con diagnóstico de enfermedad terminal en los últimos seis meses, se identificó al cuidador primario, se le invitó a participar en la encuesta sobre conocimientos de CPS y simultáneamente se identificó a pacientes terminales con necesidad de CPS hospitalizados y cuidadores de pacientes en la misma condición que acudieron a la consulta externa. Los miembros del PRACUP informaron a los sujetos de los objetivos del estudio y solicitaron su consentimiento informado, invitándolos a un área destinada para realizar la entrevista, en la que una persona no implicada en la atención del paciente entrevistaba al cuidador. Dicho personal, previamente entrenado, utilizó una encuesta diseñada para describir aspectos relevantes de los cuidados paliativos, la opinión del cuidador acerca de informar o no el diagnóstico a los pacientes o en el caso hipotético de que ellos lo fueran, además de medir el grado de sobrecarga por el cuidado. El proyecto contó con la aprobación del comité de bioética del INNN.

\section{Características del cuestionario}

Para garantizar la seguridad y fiabilidad de la información se empleó una terminología coloquial para hacer preguntas comprensibles, usándose ítems de escalas aplicadas en investigaciones realizadas sobre cuidados paliativos (14-16).

El conocimiento de cuidados paliativos se evaluó mediante dos preguntas: una valoraba si el cuidador contaba con información previa sobre CPS y la otra inquiría el significado de los CPS para el cuidador.

Las actitudes para informar y ser informado se valoraron con cinco preguntas; cuatro preguntas de respuesta cerrada y una de respuesta abierta. La primer pregunta buscaba conocer lo que el cuidador opinaba acerca de que a él se le informara con todo detalle sobre su diagnóstico y pronóstico, en el supuesto de que él fuera un paciente terminal. La segunda hacía referencia a la preferencia de la persona por quién deseaba ser informado (médico, enfermera, familiar o conocido). La tercera evaluaba el deseo del cuidador de no decirle o mentirle al paciente sobre su diagnóstico o pronóstico, en caso de que estos fuesen malos. La cuarta pregunta intentaba conocer la opinión del cuidador acerca de si se le debía informar con todo detalle sobre su diagnóstico y pronóstico a su familiar enfermo neurológico con necesidad de CPS. La quinta pregunta era abierta y pretendía conocer la razón del cuidador para optar por su respuesta en la pregunta cuatro.

En cuanto a la valoración del rol de cuidador en este proceso, se consideraron 14 ítems. Los cuales fueron agrupados en tres categorías: 1) cuidado cotidiano del paciente, 2) percepción sobre el enfermo y 3) reflexiones sobre el rol de cuidador. El cuidado cotidiano del paciente fue evaluado con indicadores relacionados con el aseo personal del paciente, la alimentación y la movilidad/reposo. Para la segunda categoría se evaluaron cuatro indicadores referidos al paciente: a) el dolor como el aspecto que más les preocupaba al cuidador; b) si consideraba que el paciente es quien tiene más difícil esta situación; c) si al paciente le gustaba recibir visitas, y d) si consideraba que la identidad del paciente seguía siendo la misma de siempre o había cambiado a partir de la enfermedad. Para 
la tercera categoría se utilizaron indicadores relacionados al cuidado cotidiano del enfermo. Estos fueron: a) la opinión del cuidador sobre si estaba preparado para afrontar y ayudar a su familiar al final de su vida; b) si consideraba su rol de cuidador como satisfactorio; c) si su familia había pasado previamente por situaciones semejantes; d) si era la única persona encargada de las actividades relacionadas con el cuidado del paciente; e) si sentía que la situación había superado sus posibilidades de hacerle frente; f) si consideraba que esta era la experiencia más difícil que había tenido que enfrentar, y g) si contaba con creencias (religiosas, espirituales, etcétera) que le apoyan para el cuidado. Las opciones de respuesta fueron cerradas (si/ no) (16).

La Escala de Zarit reducida en cuidados paliativos, consta de 7 ítems, con una calificación Likert de cinco puntos, que varía desde $1=$ nunca hasta $5=$ siempre, y su punto de corte está esta- blecido en 17 puntos (claudicación familiar $\geq 17$ puntos; sin claudicación familiar puntuaciones inferiores); proporciona una sensibilidad y especificidad en cuidados paliativos del $100 \%$, para la identificación del estado de claudicación familiar (situación de incapacidad de los miembros de la familia para ofrecer una respuesta adecuada a las múltiples demandas y necesidades del paciente). Los reactivos se agrupan en 3 factores: sobrecarga (3 ítems), autocuidado (2 ítems) y pérdida de rol social o familiar (2 ítems).

\section{Análisis estadistico}

Se realizó análisis descriptivo-comparativo (frecuencias, promedios, test estadísticos de Fisher, Mann-Whitney y Kruskal-Wallis), con un intervalo de confianza de $95 \%(p<0.05)$. Para las preguntas abiertas se aplicó un análisis de contenido, con el fin de identificar, codificar y categorizar las respuestas brindadas por el cuidador a partir de

Tabla 1. Frecuencias y porcentajes de respuestas sobre actitudes hacia informar, en cuidadores de pacientes neurológicos.

\begin{tabular}{|c|c|c|}
\hline & \multicolumn{2}{|c|}{ Respuesta } \\
\hline & $\mathrm{n}$ & $\%$ \\
\hline \multicolumn{3}{|l|}{ Si usted padeciera una enfermedad grave o incurable, ¿desearia que se lo informaran? } \\
\hline No en ningún caso. & 3 & 7,5 \\
\hline $\begin{array}{l}\text { Si con todo detalle, tanto del diagnóstico como del pronóstico aunque fueran } \\
\text { malos. }\end{array}$ & 35 & 87,5 \\
\hline $\begin{array}{l}\text { Solamente del diagnóstico, bueno o malo, pero del pronóstico solamente si es } \\
\text { bueno. }\end{array}$ & 1 & 2,5 \\
\hline Solamente del diagnóstico, bueno o malo, pero no del pronóstico sea cual sea. & 1 & 2,5 \\
\hline \multicolumn{3}{|l|}{ ¿Por quién desearía ser informado? } \\
\hline Directamente por el médico & 31 & 86,1 \\
\hline Por una enfermera & 0 & 0,0 \\
\hline A través de un familiar o una persona conocida & 5 & 13,9 \\
\hline \multicolumn{3}{|c|}{ Si el diagnóstico o el pronóstico fuesen malos para su paciente, ¿desearía que le mintiesen sobre ellos? } \\
\hline $\mathrm{Si}$ & 9 & 22,5 \\
\hline No & 31 & 77,5 \\
\hline \multicolumn{3}{|c|}{ ¿Cree que su familiar enfermo en situación de cuidados paliativos debe ser informado sobre su enfermedad? } \\
\hline No en ningún caso. & 8 & 20,0 \\
\hline $\begin{array}{l}\text { Si con todo detalle, tanto del diagnóstico como del pronóstico aunque fueran } \\
\text { malos. }\end{array}$ & 25 & 62,5 \\
\hline $\begin{array}{l}\text { Solamente del diagnóstico, bueno o malo, pero del pronóstico solamente si es } \\
\text { bueno. }\end{array}$ & 7 & 17,5 \\
\hline Solamente del diagnóstico, bueno o malo, pero no del pronóstico sea cual sea. & 0 & 0,0 \\
\hline
\end{tabular}


las respuestas obtenidas en las preguntas abiertas. Se utilizó esta técnica (análisis de contenido latente) porque resulta útil para explorar la percepción de los sujetos sobre sus experiencias y, además, limita la posibilidad de expresar hipótesis o ideas preconcebidas.

\section{Resultados}

Los diagnósticos más frecuentes de los pacientes fueron: enfermedades neurológicas con procesos neoplásicos (16), Demencias (5), Huntington (5), Enfermedad de la Neurona Motora (3), Eventos Vasculares Cerebrales (3) y Neuroinfecciones (3). La muestra presentó las siguientes características: pacientes con edad promedio de 57,9 años, con un rango de 18 a 89 años y tiempo de enfermedad de 5 años, 2 hijos en promedio, 52,6\% hombres.

Cuidadores: $77,5 \%$ mujeres, 35,0\% hijos, 25,0\% cónyuges, $15,0 \%$ padres, $12,5 \%$ hermanos y 12,5 $\%$ otros familiares. La edad fue de 49,3 años en promedio, con un rango de 17 a 80 ańos y proporcionan 18,5 horas de cuidado diario en promedio. El 62,5\% tenía estudios de secundaria o superiores. El 57,5\% de los cuidadores viven en la ciudad de México. El 40\% único cuidador, $16,7 \%$ no cuenta con redes de apoyo.

En lo referente al conocimiento sobre cuidados paliativos, 70\% de los cuidadores no tenía información previa. Con respecto al significado de los cuidados paliativos, las respuestas se agruparon en tres categorías: el 50\% mencionó "significa dar

Tabla 2. Frecuencias y porcentajes reportados por los cuidadores sobre sus experiencias de cuidado.

\begin{tabular}{|c|c|c|}
\hline \multirow[t]{2}{*}{ Experiencias del cuidado } & \multirow[b]{2}{*}{$\mathrm{n}$} & \multirow[b]{2}{*}{$\%$} \\
\hline & & \\
\hline \multicolumn{3}{|l|}{ Cuidado cotidiano del paciente } \\
\hline ¿Le insiste constantemente que coma, un poco más de lo que come? & 27 & 67,5 \\
\hline ¿Considera que asearlo y bañarlo, es la tarea más difícil al cuidarlo? & 16 & 40,0 \\
\hline ¿Le insiste al paciente a que se levante de la cama, o que sea más activo? & 27 & 67,5 \\
\hline \multicolumn{3}{|l|}{ Percepción sobre el enfermo } \\
\hline ¿Su principal preocupación es el dolor que pudiera sentir su paciente? & 32 & 80,0 \\
\hline ¿Considera que su paciente es quien tiene más difícil esta situación? & 29 & 72,5 \\
\hline ¿Al paciente le gusta recibir visitas? & 36 & 90,0 \\
\hline ¿Considera que su paciente cambió después de la enfermedad (su identidad)? & 33 & 82,5 \\
\hline \multicolumn{3}{|l|}{ Reflexiones sobre el rol de cuidador } \\
\hline ¿Cree que está preparado para afrontar y ayudar a su familiar al final de su vida? & 24 & 60,0 \\
\hline ¿Se siente satisfecho con la forma en que cumple con su papel como cuidador? & 35 & 87,5 \\
\hline ¿Su familia ha pasado antes por una situación similar? & 14 & 35,0 \\
\hline ¿Es el único cuidador? & 16 & 40,0 \\
\hline Se siente "atrapado" (sin saber qué hacer) por esta situación. & 18 & 45,0 \\
\hline ¿Considera que esta es la experiencia más difícil que ha tenido que enfrentar? & 34 & 85,0 \\
\hline Tiene creencias (religiosas, espirituales etc.) que le apoyan para el cuidado. & 30 & 75,0 \\
\hline
\end{tabular}


ayuda" (física o emocional), un $20 \%$ "es para mejorar la calidad de vida" y un 30\% dijo "no saber".

\section{A favor de informar al paciente}

Se mostró a favor de informar con detalle a su familiar enfermo $62,5 \%$ de los cuidadores. El perfil fue: mujeres (72\%), hijos (32\%), mayores de 50 años (56\%) y con alta escolaridad $56 \%$ (de 10 a 17 años). El $56 \%$ no muestra claudicación familiar y $52 \%$ son cuidadores de pacientes neurológicos.

$\mathrm{Al}$ analizar las respuestas sobre por qué informar o no informar al paciente; $25 \%$ consideró que es un derecho del paciente, $15 \%$ refirió que serviría para tomar decisiones futuras, $17,5 \%$ mencionó que evitaría el sufrimiento y $30 \%$ dio otro tipo de respuesta, "no cambia su condición, no es necesario, no entiende, se deprime, etcétera". Igualmente, al preguntarles si les gustaría que se le mintiese al paciente en caso de un diagnóstico o pronóstico malo, el 22,5\% manifestó estar de acuerdo. En la tabla 1 se muestran las frecuencias y porcentajes reportados.

Ser informado a detalle, en el caso hipotético de ser el enfermo

El $87,5 \%$ de los entrevistados, desearía ser informado con detalle si ellos fueran los pacientes, $\mathrm{Su}$ perfil: mujeres (77\%), la mayoría hijos (37\%), $51 \%$ son cuidadores mayores de 50 años, con alta escolaridad (54\%), sin claudicación familiar (63\%), 57\% son cuidadores de pacientes neurológicos.

En la tabla 2 se muestran las respuestas que dan los cuidadores sobre sus experiencias en el cuidado del paciente.

Nota. Las frecuencias y porcentajes están basadas en las respuestas afirmativas.

\section{Cuidado Cotidiano}

Referente a alimentación y actividad física, destaca que a los pacientes se les insiste en comer un poco más o ser más activos (67,5\%). Las mujeres manifiestan más esta insistencia, a diferencia de los hombres $(71,0 \%$ versus $55,6 \% ; x 2(1)=$ $0.755 ; \mathrm{p}=0.437), 75 \%$ son cuidadores jóvenes (menores de 50 años) y 60\% cuidadores mayores de 50 años $(x 2(1)=1.026 ; \mathrm{p}=0.311)$. En escolaridad: $84,2 \%$ tiene baja escolaridad (0 a 9 años) y $52,4 \%$ alta escolaridad ( 10 a 17 años) ( $x 2$ $(1)=4.607 ; p=0.046)$. El 64,7\% de cuidadores se pueden considerar con claudicación familiar y el $69,6 \%$ no la manifiesta $(x 2(1)=0.109$; $\mathrm{p}=$ $1.000)$. El $75 \%$ son cuidadores de pacientes neurológicos y $56,3 \%$ de pacientes neurooncológicos $(x 2(1)=1.538 ; \mathrm{p}=0.215)$.

\section{Percepción sobre el enfermo}

El cuidador percibe cambios en la identidad del paciente después de la enfermedad $(82,5 \%)$, esto sucede con mayor frecuencia en cuidadores con claudicación familiar, a diferencia de los que no la manifiestan $(94,1 \%$ versus $73,9 \% ; \times 2(1)=$ 2.764; $\mathrm{p}=0.205)$. El cuidador del paciente en situación terminal considera que al paciente le agrada ser visitado (90\%), aunque quienes más los visitan sean familiares $43,2 \%$, amigos y/o familiares $40,5 \%$, y solo $15 \%$ amigos.

\section{Rol del cuidador}

En cuanto a las reflexiones sobre el rol de cuidador, $87,5 \%$ está satisfecho con su papel de cuidador y un $85 \%$ de los cuidadores considera que es la experiencia más difícil que ha enfrentado.

\section{Discusión}

La muestra estudiada manifestó buena disposición a informar y ser informado, con porcentajes superiores a los reportados por Cuesta(15), lo que indica que existe sensibilidad de los cuidadores entrevistados para dar y recibir información, independientemente del pronóstico. Sin embargo, cuando el cuidador hipotéticamente asumió ser el paciente, existió una actitud contradictoria, ya que el $22,5 \%$ prefirió que se le mintiese respecto del diagnóstico o pronóstico desfavorable, lo que sugiere que probablemente la percepción real de algunos cuidadores respecto del tema sigue siendo conservadora y mantiene patrones culturales en los que el enfermo es dependiente de terceros, no tiene un papel activo en la toma de decisiones y prefiere la actitudes paternalistas por parte de los médicos. 
A pesar del estrés y sobrecarga, los entrevistados expresaron sentirse satisfechos en su papel de cuidadores; no obstante, es necesario explorar qué factores son y deben ser potencializados para sobrellevar los cuidados y la etapa terminal del paciente neurológico, mediante estudios controlados, muestras más amplias y comparando diferentes grupos de padecimientos. Una debilidad de la presente investigación es el reducido tamaño de la muestra y la heterogeneidad de los padecimientos neurológicos, limitantes para realizar generalizaciones más amplias. No obstante las características de la muestra, el estudio es pionero en México, tanto por abordar padecimientos neurológicos en situación de terminalidad, como por elegir a los cuidadores como población de estudio y reportar la percepción de su rol de cuidadores.

Es indudable que la atención paliativa se brinda en momentos muy difíciles para la persona enferma, su cuidador y sus familiares, en los que las actitudes y sentimientos pueden generar disonancia emocional que impida una buena comunicación y eficacia en la atención terminal.
En esta población, en la que el canal preferente de información y de confianza sigue siendo el médico, el personal de salud debe formarse para desarrollar habilidades y competencias que permitan brindar información y atención, tanto al paciente como al cuidador, con un enfoque bioético.

\section{Conclusión}

En este trabajo se reportan datos que permiten sugerir una tendencia - marcada por generación, sexo, escolaridad y cercanía de la relación-, consistente en una mayor sensibilidad sobre informar y recibir información del diagnóstico y pronóstico de padecimientos terminales.

El presente estudio evidencia la necesidad de informar ampliamente sobre el propósito de los cuidados paliativos a cuidadores de pacientes con alguna enfermedad neurológica, ya que en la muestra encuestada un alto porcentaje no tenía información previa sobre los cuidados paliativos y el 30\% no supo decir el significado de los mismos, lo que concuerda con lo que previamente hemos dicho acerca de la insuficiencia en la formación de recursos humanos y de programas dirigidos a atender dicha necesidad en pacientes neurológicos en México. 
Estudio piloto en cuidadores de pacientes con enfermedades neurológicas - Zoila Trujillo de los Santos et al.

\section{Referencias}

1. León-Delgado M, Flórez-Rojas S, Torres M, Rengifo-Varona M, Prada D. La importancia de evaluar síntomas y alteraciones funcionales en enfermedades neurológicas crónicas: experiencia en cuidado paliativo y rehabilitación en una institución colombiana. Aquichan 2010; 10(3): 228-243.

2. Jiménez OM, Camargo LMR. Diagnóstico del Servicio de Cuidados Paliativos. México: Hospital General de México, Secretaria de Salud; 2013.

3. González C, Méndez J, Romero JI, et al. Cuidados paliativos en México. Revista Médica Hospital General de México 2012; 75(3): 173-179.

4. The Lancet Oncology Comisión. La planificación del control del cáncer en América Latina y el Caribe. The Lancet Oncology 2013; 14: 1-52.

5. World Health Organization Cancer Pain Relief and Palliative: a report of OMS expert committee. Geneva: World Health Organization; 1990.

6. Cámara de Diputados, Honorable Congreso de la Nación. Ley General de Salud en Materia de Cuidados Paliativos. Última Reforma Publicada Diario Oficial de la Federación 01/V/2009.

7. Paz O. El Ogro Filantrópico. México, D.F.: Joaquín Mortiz; 1979: 85.

8. Dieterlen P. Paternalismo y Estado de Bienestar. Doxa 1988; 5: 175-194.

9. Azula A. Los principios bioéticos: ¿̨se aplican en la situación de enfermedad terminal? Anales Medicina Interna (Madrid) 2001; 18: 650-654.

10. Gillon R. Paternalism and medical ethics. British Medicine Journal 1985; 290: 1971.

11. Loignon C, Boudreault-Fournier A. From paternalism to benevolent coaching. New model of care. Canadian Family Physician 2012; 58: 1194-1195.

12. Paz-Rodríguez F, Sánchez-Guzmán MA. Identificación de variables predictoras de violencia en díadas paciente-cuidador de cuatro enfermedades neurológicas. Revista Mexicana Neurociencias 2012; 13(4): 205-214.

13. Paz-Rodríguez F, Sánchez-Guzmán MA. Maltrato en pacientes neurológicos. Anales de Psicología 2011; 27(1): 256-264.

14. Luxardo N, Tripodoro B, Funes M, et al. Perfil de cuidadores de enfermos en cuidados paliativos. Medicina (Buenos Aires) 2009; 69: 519-525.

15. Cuesta GJ, Martínez MA, Rodríguez HA. Informar o no informar a los pacientes paliativos: ¿qué opinan sus familiares? Medicina Paliativa 2012; 19(2): 58-63.

16. Gort A M, March J, Gómez X, et al. Escala de Zarit reducida en cuidados paliativos. Medicina Clínica (Barcelona) 2005; 124(17): 651-653.

Recibido: 8 de abril de 2014

Aceptado: 28 de mayo de 2014 